\title{
Tratamiento metalúrgico de los óxidos Waelz mediante lixiviación alcalina utilizando carbonato amónico
}

\author{
V. Meseguer ${ }^{(*)}$, L.J. Lozano ${ }^{(*)}$ y D. de Juan ${ }^{(*)}$
}

\begin{abstract}
Resumen Se propone un método para el tratamiento de los óxidos Waelz, y se estudian, a nivel de laboratorio, las principales etapas implicadas en el mismo. El proceso consiste básicamente en la lixiviación de los óxidos con una disolución de carbonato amónico/amoniaco, y posterior recuperación del cinc lixiviado mediante su precipitación como carbonato básico de cinc. Las etapas que constituyen el proceso y que han sido objeto de estudio son: lixiviación de los óxidos Waelz, purificación de la lejía concentrada de lixiviación, precipitación del cinc, lavado del carbonato básico de cinc precipitado y transformación del carbonato básico de cinc. El tratamiento diseñado presenta un impacto ambiental prácticamente nulo, y con el mismo se puede recuperar más del $90 \%$ del cinc contenido en los óxidos Waelz, así como el $80 \%$ del cobre y del cadmio. Además, el proceso presenta gran versatilidad en cuanto que el cinc se puede recuperar én el estado más conveniente (cinc metal, óxido o sal).
\end{abstract}

Palabras clave: Óxidos Waelz. Tratamiento de residuos. Lixiviación. Recuperación de cinc. Carbonato amónico.

\section{Metallurgical treatment of Waelz oxides by alkaline leaching using ammonium carbonate}

\begin{abstract}
A method to carry out the treatment of the Waelz oxides is proposed, and the principal stages implicated are studied laboratory-scale. The process consists of the oxides leaching with an ammonium carbonate/ammonia solution, followed the recovery of the zinc leached by means of its precipitation as zinc basic carbonate. The process stages that have been studied are: Waelz oxides leaching, loaded leach purification, zinc precipitation, zinc basic carbonate washing and zinc basic carbonate transformation. The designed treatment presents a null environmental impact, and more than $90 \%$ of the zinc contained in the Waelz oxides, as well as $80 \%$ of the copper and cadmium contents could be recovered. Also, the process presents great flexibility as soon as zinc could be recovered in the most convenient form (zinc metal, oxide or salt).
\end{abstract}

Keywords: Waelz oxides. Waste treatment. Leaching. Zinc recovery. Ammonium carbonate.

\section{INTRODUCCIÓN}

Los óxidos Waelz se producen en el tratamiento al que se someten los residuos polimetálicos y las materias primas naturales de baja ley al objeto de recuperar los metales volátiles, fundamentalmente cinc, plomo y cadmio, presentes en los mismos.

(•) Trabajo recibido el día 12 de julio de 1996.

(*) Grupo de Investigación INQUICA. Dpto. de Ingeniería Química Cartagena. Universidad de Murcia. Paseo Alfonso XIII, 34. 30203-Cartagena, Murcia. (España)
Por su composición polimetálica y elevado contenido de cloruros (Tabla I), estos óxidos son difíciles de tratar siguiendo técnicas metalúrgicas clásicas, ya sean hidro o pirometalúrgicas. Actualmente, los óxidos Waelz se tratan en el Imperial Smelting Furnace (ISF), aunque la cantidad procesada está limitada a un $10-15 \%$ de la carga total del horno (1).

Por otra parte, la deposición, controlada o no, de los polvos volantes del horno eléctrico de arco (EAF), está planteando un problema medioambiental, ya que estos productos, por sus propiedades 
TABLA I.- Composición química media de los óxidos Waelz

TABLE I.-Average chemical composition of Waelz oxides

\begin{tabular}{|c|r|c|c|c|c|c|c|}
\hline Elemento & $\%$ & Elemento & $\%$ & Elemento & $\%$ & Elemento & $\%$ \\
\hline $\mathrm{Zn}$ & 56,00 & $\mathrm{Sn}$ & 0,40 & $\mathrm{C}$ & 0,80 & $\mathrm{MgO}$ & 0,50 \\
$\mathrm{Fe}$ & 3,00 & $\mathrm{As}$ & 0,02 & $\mathrm{Mn}$ & 0,60 & $\mathrm{BaO}$ & 0,01 \\
$\mathrm{~Pb}$ & 11,00 & $\mathrm{~S}$ total & 1,80 & $\mathrm{Na}$ & 1,90 & $\mathrm{Al}_{2} \mathrm{O}_{3}$ & 0,20 \\
$\mathrm{Cu}$ & 0,04 & $\mathrm{~F}$ & 0,60 & $\mathrm{~K}$ & 2,10 & $\mathrm{SiO}_{2}$ & 0,70 \\
$\mathrm{Cd}$ & 0,20 & $\mathrm{Cl}$ & 4,00 & $\mathrm{CaO}$ & 0,80 & & \\
\hline
\end{tabular}

físicas y químicas, se pueden caracterizar como Residuos Tóxicos y Peligrosos (RTP's).

$\mathrm{La}$ industria siderúrgica produce unos $15 \mathrm{~kg}$ de polvos de EAF por tonelada de acero fabricado, lo que supone 115.000 t/año en España y 700.000800.000 t/año en la CE. En España sólo se trata un $20 \%$ de esta cantidad, y el resto se almacena en escombreras de difícil control.

Debido al problema que presenta la acumulación de estos polvos de EAF, se están haciendo múltiples intentos para llevar a cabo su tratamiento, tanto por vía térmica como por vía húmeda. Entre los primeros se pueden citar los métodos que utilizan las técnicas de plasma (2 y 3 ) y el proceso St. Joe (4), aunque, por ahora, y en Europa, la técnica más extendida es el proceso Waelz (5). También se han desarrollado diversos procesos para tratar dichos productos por vía húmeda, tales como el proceso CENIM-LNETI (6) o el desarrollado por Técnicas Reunidas, aunque ninguno de ellos ha alcanzado éxito comercial.

Como se ha indicado, en Europa se está apostando por realizar el tratamiento de los polvos de EAF siguiendo el proceso Waelz, lo que conlleva la aparición de un nuevo problema: la generación de óxidos Waelz. Puesto que la cantidad de óxidos Waelz que se pueden tratar en el ISF es limitada, las quince unidades ISF existentes en el mundo sólo pueden admitir en su carga $2 \cdot 10^{5}-3 \cdot 10^{5}$ t/año de óxidos Waelz. Este consumo equivale a los óxidos Waelz que se producirían en el tratamiento total, a través del horno Waelz, de los polvos volantes generados en las acerías europeas, por lo que no sería posible tratar el resto de los polvos de EAF mundiales.

Es preciso, por tanto, encontrar caminos alternativos que permitan el tratamiento directo de los polvos de EAF o de los óxidos Waelz producidos en su procesamiento. Puesto que el tratamiento directo de los polvos de EAF no es fácil, parece más factible diseñar un método para tratar los óxidos Waelz que solucione indirectamente el problema planteado.

Como alternativas pirometalúrgicas al tratamiento de los óxidos Waelz en el ISF, se pueden mencionar los métodos derivados de la técnica del plasma (2 y 3) o del horno KIVCET (7). Estas técnicas están en desarrollo y presentan costes elevados, tanto de inversión como energéticos.

Las alternativas hidrometalúrgicas son muy variadas, aunque existen diversas dificultades de proceso para su aplicación práctica. Los métodos clásicos, basados en la lixiviación sulfúrica y posterior electrólisis, se encuentran con el problema que plantea el alto contenido en haluros, fundamentalmente cloruros, que poseen los óxidos Waelz.

Otros métodos basados en la lixiviación ácida, cómo, por ejemplo, la lixiviación clorhídrica, pasan por la electrólisis de cloruros (8) o por técnicas de extracción con disolventes (9) para realizar un cambio de anión, o emplean una solución mixta extracción/electrólisis de cloruros (10). Aunque se han realizado diversos intentos para efectuar la electrólisis de cloruros (11), ninguno ha alcanzado éxito comercial.

La extracción con disolventes orgánicos es relativamente costosa; se emplea comercialmente en la recuperación de cinc a partir de disoluciones de cinc con otros cationes que pueden proceder de materiales residuales sin valor. En principio, en el estado actual de la técnica, la aplicación de este método a una materia prima con valor económico no es rentable.

Los métodos alcalinos, como el proceso CENIM-LNETI (6), parecen ofrecer vías alternativas con ciertas posibilidades de éxito. En este proceso se plantean diversos problemas, tales como:

- La utilización como agente lixiviante de una disolución de cloruro amónico/amoniaco incorpora gran cantidad de anión cloruro, cuya presencia es totalmente nociva en una electrólisis clásica de cinc.

- Se emplea la extracción con disolventes orgánicos para realizar el cambio de anión acompañante, con el coste adicional que ello supone.

- El cinc recuperado sólo se puede ofertar al mercado en estado metálico o como sulfato.

Por último, parece claro que sólo será posible aplicar un tratamiento especial a una materia prima de alto coste cuando el mismo tenga la suficiente versatilidad como para poder ofertar al mercado el 
producto que posea en cada momento el mayor valor añadido.

Llegados a este punto, el objetivo del presente trabajo es desarrollar un proceso hidrometalúrgico alternativo para llevar a cabo el tratamiento de los óxidos Waelz.

\section{PROPUESTA DE UN MÉTODO ALTERNATIVO}

Teniendo en cuenta lo indicado anteriormente, el método propuesto debe contemplar los siguientes condicionantes:

- No introducir aniones nocivos en una electrólisis de cinc.

- Versatilidad en cuanto a la obtención del producto final.

- Presentar el mínimo impacto ambiental.

En este sentido, en un trabajo previo (12), se desarrolló un método para realizar la lixiviación de los óxidos Waelz. Se encontró que las condiciones óptimas se daban al emplear como agente de lixiviación una disolución $2,5 \mathrm{M}$ en carbonato amónico de $\mathrm{pH} 10,0-10,5$, el cual se conseguía por adición de una disolución acuosa de amoniaco al $25 \%$ en volumen.

En la disolución resultante de la lixiviación el cinc se encuentra como $\mathrm{CO}_{3}\left[\mathrm{Zn}\left(\mathrm{NH}_{3}\right)_{4}\right]$ (13). Puesto que es deseable obtener el cinc en estado de carbonato, óxido o hidróxido, será preciso:

- Eliminar el amoniaco libre de la disolución.

- Descomponer el complejo de cinc.

Para conseguir ambos propósitos se pueden seguir dos vías:

- Neutralización con un agente ácido, tal como el $\mathrm{CO}_{2}$.

- Descomposición hidrotermal y eliminación de los productos resultantes, $\mathrm{CO}_{2}$ y $\mathrm{NH}_{3}$, mediante su arrastre con otro gas.

Ambos sistemas presentan ventajas e inconvenientes. A primera vista, el método más conveniente, aunque más complejo, parece ser el segundo, del cual existen datos referidos fundamentalmente al níquel (10). Este método de actuación presenta la ventaja de que permite obtener como producto un carbonato básico de cinc no estequiométrico, que puede transformarse posteriormente, de manera fácil, en el producto de cinc (sal, óxido o metal) que presente mayor valor comercial.

Puesto que para obtener un producto económicamente valorable es necesario que tenga la máxima pureza posible, será preciso depurar las disoluciones antes de proceder a la precipitación del compuesto de cinc.
De acuerdo con estas premisas, se ha planteado, para realizar el tratamiento de los óxidos Waelz, el proceso esquematizado en la figura 1. En esencia, el proceso desarrollado consiste en la lixiviación de los óxidos Waelz con una disolución de carbonato amónico/amoniaco y posterior recuperación del cinc lixiviado mediante su precipitación como carbonato básico de cinc, con las operaciones auxiliares precisas para conseguir la calidad necesaria en el producto final.

Se han estudiado en el laboratorio las principales etapas implicadas en este proceso, es decir:

- Lixiviación de los óxidos Waelz.

- Purificación de la lejía concentrada de lixiviación.

- Precipitación del cinc.

- Lavado del carbonato básico de cinc.

- Transformación del carbonato básico de cinc.

\section{DESARROLLO EXPERIMENTAL}

\subsection{Lixiviación de los óxidos Waelz}

\subsubsection{Método operatorio}

La lixiviación se ha llevado a cabo en dos etapas a contracorriente, simulando un proceso en continuo, tal y como se muestra en la figura 2.

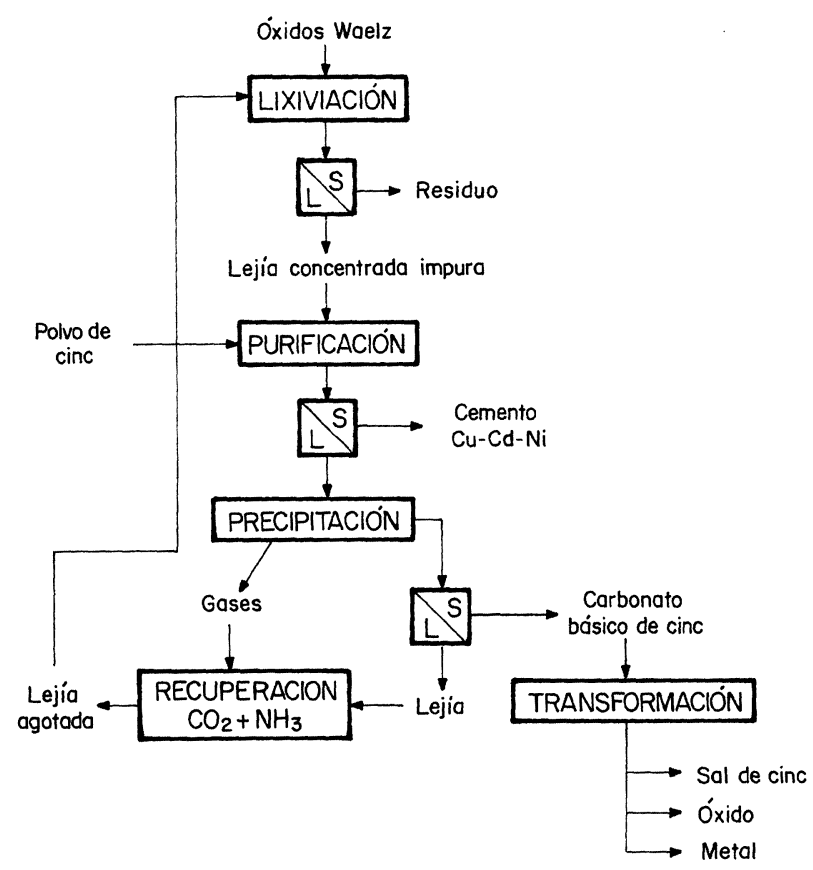

FIG. 1.- Proceso propuesto inicialmente para el tratamiento de los óxidos Waelz.

FIG. 1.- Initial process proposed for the Waelz oxides treatment. 


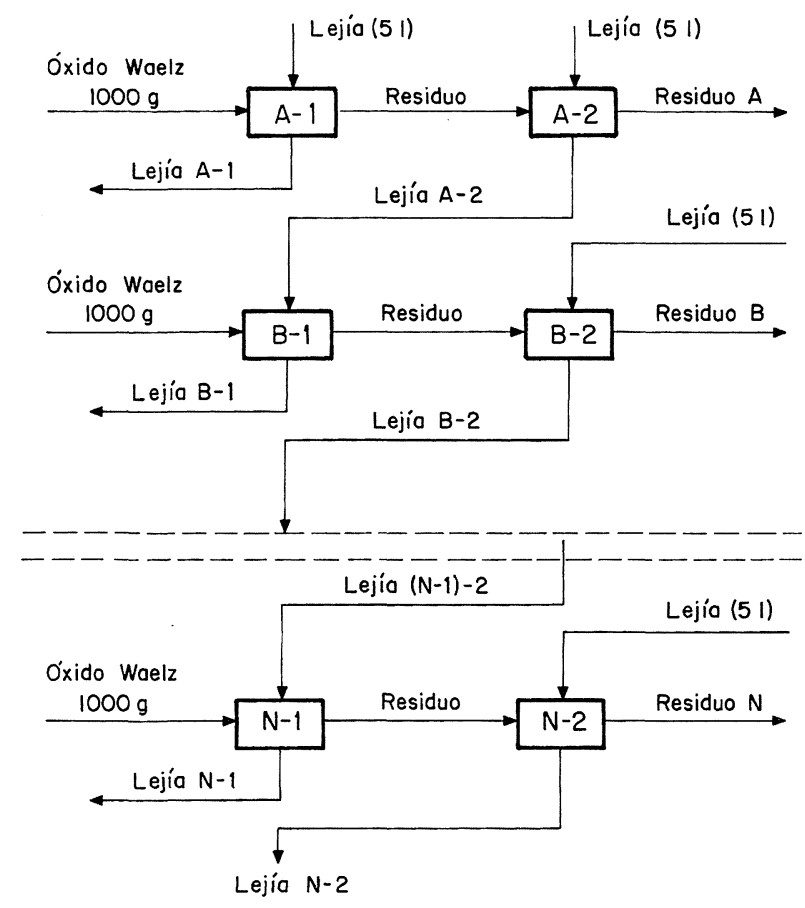

FIG. 2.- Tratamiento de lixiviación de los óxidos Waelz.

FIG. 2.-Leaching treatment of Waelz oxides.

Los óxidos Waelz empleados en este trabajo han sido suministrados por Aser, S.A., y proceden del tratamiento de polvos volantes de acería. Su composición química se recoge en la tabla II. Como lejía de ataque se utilizó una disolución de carbonato amónico 2,5 M, cuyo pH se situó entre 10,1-10,5 mediante la adición de una disolución acuosa de amoniaco al $25 \%$. La lixiviación se realizó con agitación mecánica, a temperatura ambiente, durante $90 \mathrm{~min}$. La relación sólido/líquido inicial fue de 1/5 (200 g de óxidos Waelz por litro de disolución lixiviante).

Finalizada la operación de lixiviación, se procedió a una separación de fases sólido/líquido mediante sedimentación. Este proceso se favoreció por el empleo de un floculante de tipo catiónico, Nalco N-70 o similar. La relación de espesamiento, definida como el cociente entre las concentraciones ( $\mathrm{g} / \mathrm{l})$ de sólido en la pulpa de salida y en la alimentación, al adicionar un $1 \%$ en volumen de disolución Nalco N-70 al 0,3\%, estaba comprendida entre 8 y 10.

Los lodos procedentes de la separación por decantación se filtraron a vacío en un embudo Buchner. La lejía de filtración se incorporó a la utilizada en la primera etapa de lixiviación para realizar el ataque, y el residuo sólido se desecó en estufa a $105^{\circ} \mathrm{C}$.

\subsubsection{Resultados obtenidos}

Los resultados obtenidos en el proceso de lixiviación se recogen en las tablas III y IV. Como se aprecia, se consiguió recuperar la mayor parte del cinc presente en los óxidos Waelz, aproximadamente el $94 \%$, así como gran cantidad de cobre y de cadmio. Por otra parte, el residuo de la lixiviación, único producto indeseable del proceso desde el punto de

TABLA II.- Composición analítica (\%) de los óxidos Waelz utilizados

TABLE II.- Elemental composition (\%) of utilized Waelz Oxides

\begin{tabular}{|llr|}
\hline \multirow{2}{*}{ CINC } & Total & 52,33 \\
& Soluble en agua & 0,84 \\
& Soluble en disolución ácida $(\mathrm{pH} 2)$ & 51,76 \\
\hline \multirow{2}{*}{ HIERRO } & Total & 5,69 \\
& Soluble en disolución ácida $(\mathrm{pH} 2)$ & 0,78 \\
\hline \multirow{2}{*}{ COBRE } & Total & 0,33 \\
& Soluble en disolución ácida $(\mathrm{pH} 2)$ & 0,32 \\
\hline CADMIO & Total & 0,29 \\
& Soluble en disolución ácida $(\mathrm{pH} 2)$ & 0,28 \\
\hline PLOMO & Total & 8,25 \\
\hline INSOLUBLES & & 2,07 \\
\hline $\mathrm{CaO}$ & & 1,92 \\
\hline $\mathrm{MgO}$ & & 0,80 \\
\hline $\mathrm{Al} \mathrm{O}_{3}$ & & 0,36 \\
\hline $\mathrm{Cl}$ & & 2,21 \\
\hline
\end{tabular}


TABLA III.- Productos obtenidos en la lixiviación de los óxidos Waelz

TABLE III.- Products obtained from the Waelz oxides leaching

\begin{tabular}{|c|c|c|c|c|c|c|c|c|}
\hline \multirow[t]{2}{*}{ Producto } & \multirow[t]{2}{*}{ Cantidad } & \multicolumn{7}{|c|}{ Composición, \% o g/l } \\
\hline & & $\mathrm{Zn}$ & $\mathrm{Fe}$ & $\mathrm{Pb}$ & $\mathrm{Cu}$ & $\mathrm{Cd}$ & $\mathrm{Cl}$ & $\mathrm{NH}_{3}$ libre \\
\hline Óxido Waelz & $100 \mathrm{~g}$ & 52,30 & 5,70 & 8,30 & 0,30 & 0,29 & 2,21 & - \\
\hline Lejía rica en cinc & $516 \mathrm{ml}$ & 95,00 & 0,35 & 0,36 & 0,45 & 0,47 & 3,85 & - \\
\hline Aguas de lavado & $52 \mathrm{ml}$ & 9,60 & 0,03 & 0,16 & 0,06 & 0,04 & 0,90 & 4,40 \\
\hline Residuo & $28,7 \mathrm{~g}$ & 9,80 & 19,80 & 28,50 & 0,25 & 0,16 & 0,75 & - \\
\hline
\end{tabular}

TABLA IV.- Rendimientos de lixiviación

TABLE IV.-Leaching performance

\begin{tabular}{|l|cccccc|}
\hline Elemento & $\mathrm{Zn}$ & $\mathrm{Fe}$ & $\mathrm{Pb}$ & $\mathrm{Cu}$ & $\mathrm{Cd}$ & $\mathrm{Cl}$ \\
\% recuperado & 93,7 & 3,17 & 2,2 & 77,4 & 83,6 & 89,9 \\
\hline
\end{tabular}

vista medioambiental, presenta un contenido de plomo que hace posible su empleo como materia prima en una metalurgia convencional de este metal, aunque sea con valor comercial de intercambio igual a cero. De esta forma, este producto deja de ser un residuo para convertirse en una materia prima y, por lo tanto, es prácticamente inocuo desde el punto de vista medioambiental. Asimismo, se puede comprender la dificultad que cualquier hidrometalurgia clásica encuentra para el tratamiento de este material, dado el alto contenido de cloro soluble.

\subsection{Purificación de la lejía concentrada}

\subsubsection{Método operatorio}

Si se pretende obtener un producto de cinc de elevada calidad será preciso, en primer lugar, eliminar de la disolución resultante de la lixiviación aquellos metales que puedan coprecipitar con el carbonato básico de cinc, tales como $\mathrm{Cu}, \mathrm{Cd}$ y $\mathrm{Pb}$. Se consideró que la mejor forma de realizar este proceso era mediante cementación con polvo de cinc.
La cementación se llevó a cabo en vaso abierto, con agitación mecánica y a temperatura ambiente. Para ello se adicionó en primer lugar un $50 \%$ de polvo de cinc en exceso respecto del estequiométrico necesario para proceder a la cementación del cobre y del cadmio. Tras 30 min de agitación, se determinó el cobre residual presente en la lejía, comprobándose que su concentración era superior a los $5 \mathrm{mg} / \mathrm{l}$. Para completar el proceso de depuración se agregó un $20 \%$ más de polvo de cinc, y después de 15 min de agitación la concentración residual de cobre era inferior a $5 \mathrm{mg} / \mathrm{l}$. En este punto se dio por finalizado el proceso, y se procedió a la separación sólido/líquido mediante filtración a vacío.

\subsubsection{Resultados obtenidos}

Los resultados obtenidos en la etapa de purificación se presentan en la tabla V. Se puede indicar que la cementación con polvo de cinc permite eliminar de la disolución prácticamente la totalidad del cobre, el plomo y el cadmio, mientras que el hierro continúa mayoritariamente en la disolución $(75 \%)$.

Como era de esperar, la concentración de ion cloruro en la lejía no se modifica, lo que obligará posteriormente a realizar una purga controlada con el fin de mantener dicha concentración en un valor correcto.

Se obtiene, en definitiva, un cemento muy interesante para su empleo como materia prima en el

TABLA V.- Resultados obtenidos en la etapa de purificación de la lejía rica

TABLE V.- Results obtained in the loaded leach purification stage

\begin{tabular}{|c|c|c|c|c|c|c|c|c|}
\hline \multirow[t]{2}{*}{ Producto } & \multirow[t]{2}{*}{ Cantidad } & \multicolumn{7}{|c|}{ Composición, \% o g/l } \\
\hline & & $\mathrm{Zn}$ & $\mathrm{Fe}$ & $\mathrm{Pb}$ & $\mathrm{Cu}$ & $\mathrm{Cd}$ & $\mathrm{Cl}$ & Insol. \\
\hline $\begin{array}{l}\text { Alimentación } \\
\text { Lejía rica en cinc }\end{array}$ & $1 \mathrm{~L}$ & 95 & 0,35 & 0,36 & 0,45 & 0,47 & 3,85 & - \\
\hline Polvo de cinc total & $1,35 \mathrm{~g}$ & 99 & - & - & - & - & - & - \\
\hline $\begin{array}{l}\text { Productos } \\
\text { Leiía rica purificada }\end{array}$ & $1 \mathrm{~L}$ & 96 & 0.26 & $<0,005$ & $<0,005$ & $<0,005$ & 3.85 & - \\
\hline Cemento & $1,50 \mathrm{~g}$ & 2,0 & 6,0 & 23,7 & 30,0 & 31,3 & 0,10 & 6,5 \\
\hline
\end{tabular}


proceso COCADEX (14), con lo que se minimiza su posible impacto ambiental.

\subsection{Recuperación del cinc presente en la lejía de lixiviación purificada}

\subsubsection{Método operatorio}

Esta etapa, básica en el proceso en estudio, ha sido ampliamente investigada y desarrollada en el caso del níquel. En el caso que nos ocupa, se plantean ciertos inconvenientes, tales como la presencia de hierro y cloro en la lejía purificada.

El método seguido para la precipitación de un compuesto básico de cinc se basa en la eliminación del amoniaco libre y posterior descomposición del carbonato precipitado mediante un tratamiento térmico a baja temperatura.

El proceso se llevó a cabo en un reactor ciclónico, de 2 L de capacidad, calentado mediante una resistencia eléctrica sumergida y provisto de control de temperatura. En algunos ensayos también se empleó, como agente de calefacción, vapor de agua saturado a 1,1 atm. En la parte inferior del reactor se incorporó una tobera por la que se introdujo aire cuando la calefacción se realizó eléctricamente, o vapor de agua cuando éste se utilizó como agente calefactor. Esta corriente gaseosa se empleó como gas de arrastre para facilitar la expulsión de los gases procedentes de la lejía de lixiviación. La instalación del reactor se llevó a cabo de forma que era posible controlar la presión de trabajo así como recuperar el líquido condensado y los vapores desprendidos, tal y como se muestra en la figura 3.

Los ensayos experimentales se realizaron siguiendo la siguiente metodología:

- Llenar el reactor ciclónico con la lejía de trabajo y fijar las condiciones de presión y temperatura.

- Una vez alcanzada la temperatura de trabajo, controlar el tiempo y, a partir del instante en que comenzaba la precipitación, tomar periódicamente muestras de la lejía para su análisis.

Siguiendo esta metodología se estudió el efecto que ejercen diversas variables del proceso, tales como temperatura y presión de operación, sobre el desarrollo del mismo.

\subsubsection{Resultados obtenidos}

\subsubsection{Punto de ebullición de las lejías}

Dado que puede ser de interés conocer las condiciones de presión y temperatura a las que se

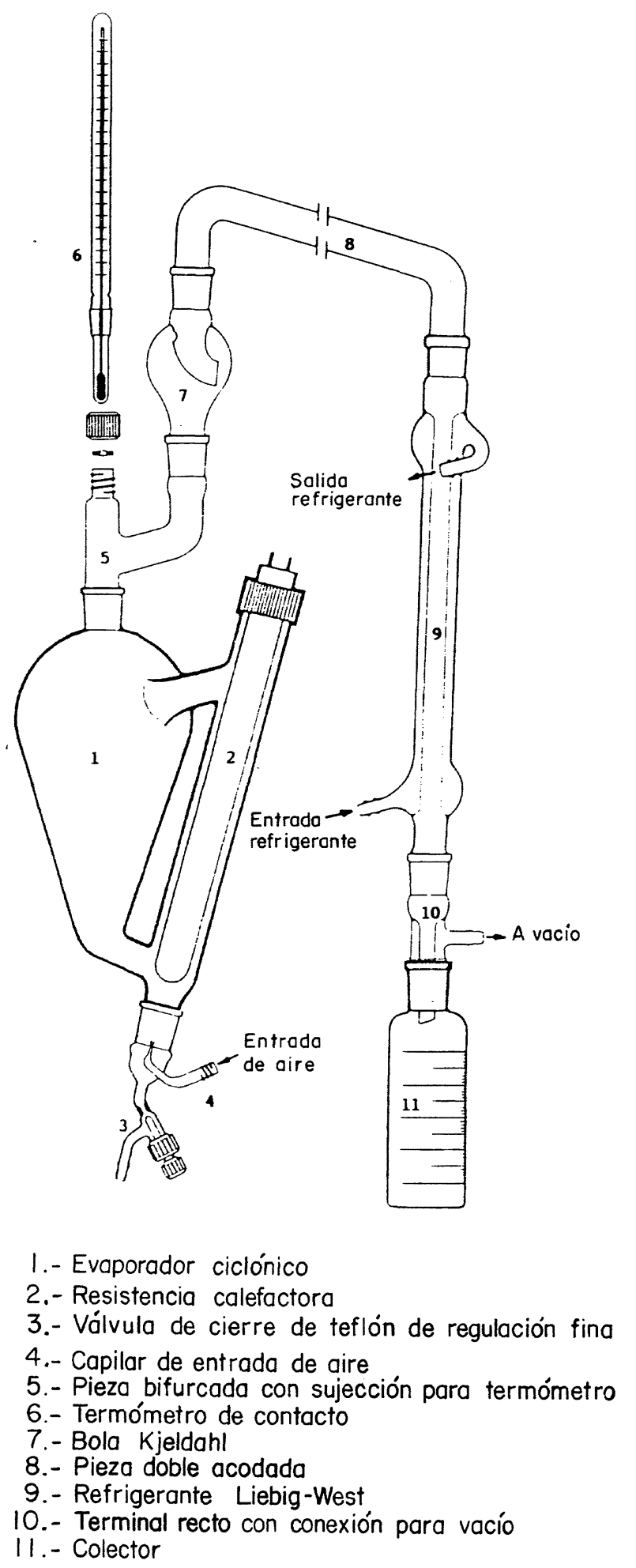

FIG. 3.- Equipo utilizado para la precipitación del carbonato básico de cinc.

FIG. 3.- Equipment used for the zinc basic carbonate precipitation.

encuentran en ebullición las lejías procedentes de la lixiviación, inicialmente se determinó la temperatura de ebullición de estas lejías en función de la presión de trabajo. 
La disolución se calentó hasta una temperatura determinada y, una vez estabilizada la misma, se aplicó vacío en el reactor hasta que comenzó la ebullición. Determinado este punto, se volvía a la presión atmosférica, se calentaba hasta otra temperatura y se repetía la operación. De esta forma se obtuvo la temperatura de ebullición de la disolución en función de la presión de trabajo.

$\mathrm{Al}$ hacer un análisis de regresión de los valores determinados se encontró que entre la presión y la temperatura existe una relación del tipo

$$
P=A+B \cdot \frac{10^{3}}{T}
$$

donde $P$ es la presión absoluta expresada en atmósferas y $T$ la temperatura en Kelvin. Para las lejías estudiadas, los valores de $A$ y $B$ son, respectivamente, 5,76 y $-1,83$.

Se debe entender que la relación encontrada es meramente orientativa, ya que depende, entre otros parámetros, de la concentración de la disolución y de la cantidad de amoniaco libre que contenga, los cuales variarán dentro de unos límites.

\subsubsection{Efecto de la temperatura y de la presión de trabajo}

Se ha estudiado la influencia de la temperatura y de la presión de trabajo sobre el proceso de precipitación del carbonato básico de cinc. Para ello se realizaron ensayos a diversas temperaturas y a dos presiones diferentes.

Como se aprecia en la figura 4, la velocidad con que precipita el carbonato aumenta al incrementarse la temperatura, y tiende a un valor máximo para la temperatura de ebullición de la disolución.

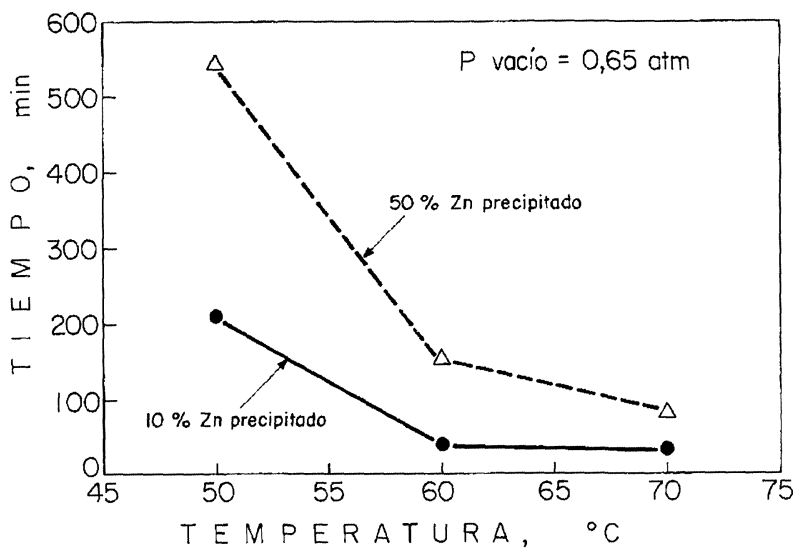

FIG. 4.- Efecto de la temperatura sobre la velocidad de precipitación del cinc.

FIG. 4.- Temperature influence over zinc precipitation velocity.
En las figuras 5 y 6 se observa que al aumentar la temperatura de operación se produce un incremento de los restantes parámetros estudiados, eliminación de amonio, precipitación de cinc y eliminación de agua, trabajando a 0,65 atm de vacío y empleando como gas de arrastre tanto aire como vapor de agua saturado a 1,1 atm.

En la figura 7 se recogen los resultados determinados en función de la presión de trabajo. Se aprecia que al disminuir la presión de trabajo en el reactor (aumentar la presión de vacío), las velocidades de eliminación de agua y de amonio así como la velocidad de precipitación del cinc tienden a aumentar. Al analizar el amonio existente en los distintos puntos de control se ha comprobado que existe una pérdida que oscila entre el 5 y el $10 \%$.

En todos los casos se observa que, tras una primera etapa que dura entre 10 y $15 \mathrm{~min}$ a partir del momento en que se alcanza la temperatura y presión de trabajo (en los ensayos realizados en las condiciones consideradas como óptimas), la eliminación de $\mathrm{NH}_{4}^{+}$sigue un camino paralelo a la precipitación del'cinc.

A este respecto se puede considerar que, inicialmente, se produce la eliminación del amoniaco libre existente en la disolución y, a partir de ese instante, tienen lugar simultáneamente la precipitación del cinc y la eliminación de amonio.

Esta mayor velocidad de eliminación de amonio en los primeros momentos del proceso da lugar a que su velocidad media de eliminación sea ligeramente superior a la del cinc, llegando a alcanzar un valor de $1,6 \mathrm{~g} / \mathrm{l} \cdot \mathrm{min}$ si se inyecta vapor saturado como agente de arrastre y calefacción.

En la recuperación del amoniaco de los gases mediante condensación y absorción, se produce la

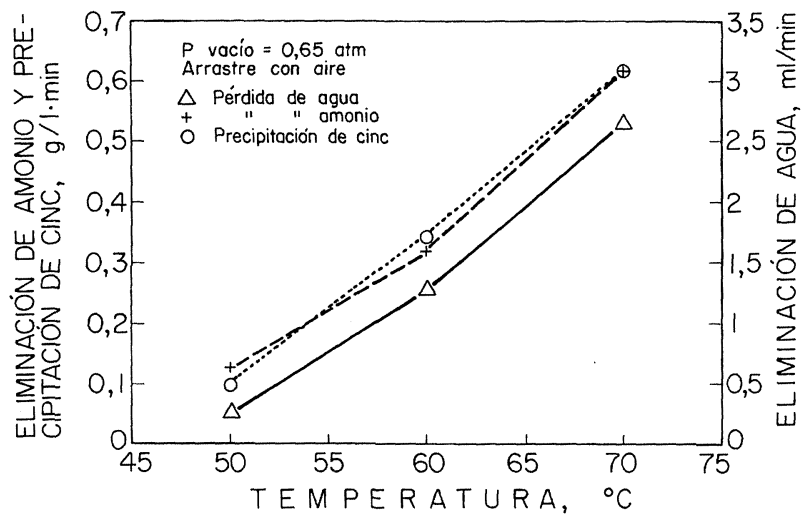

FIG. 5.- Efecto de la temperatura sobre la velocidad de eliminación de amonio y de agua y sobre la velocidad de precipitación del cinc empleando arrastre con aire.

FIG. 5.- Temperature influence over ammonium and water elimination velocity and over zinc precipitation velocity using air dragging. 


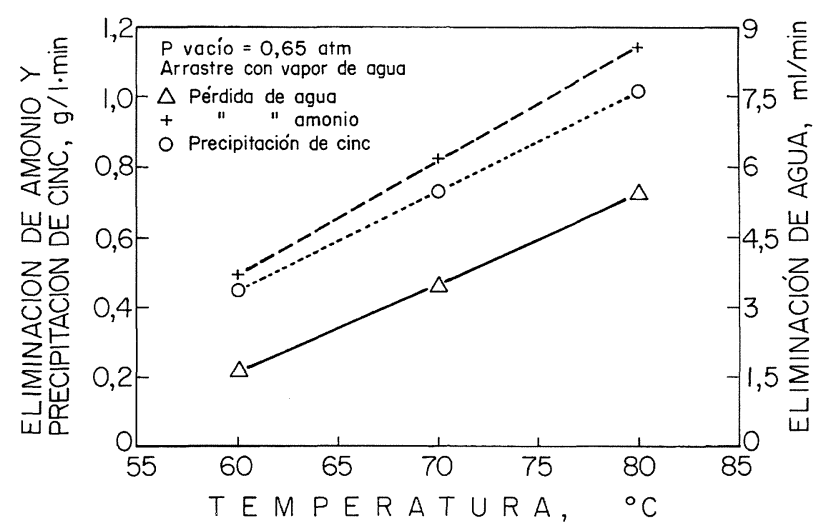

FIG. 6.- Efecto de la temperatura sobre la velocidad de eliminación de amonio y de agua y sobre la velocidad de precipitación del cinc empleando arrastre con vapor.

FIG. 6.- Temperature influence over ammonium and water elimination velocity and over zinc precipitation velocity using steam dragging.

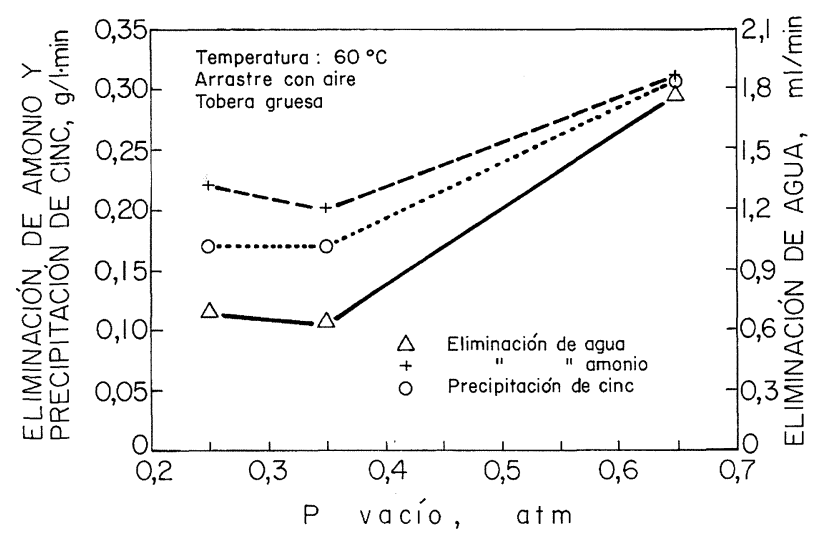

FIG. 7.- Efecto de la presión sobre la velocidad de eliminación de amonio y de agua y sobre la velocidad de precipitación del cinc.

FIG. 7.- Pressure influence over ammonium and water elimination velocity and over zinc precipitation velocity.

reacción de recombinación del $\mathrm{CO}_{2} \mathrm{y} \mathrm{NH}_{3}$, proceso fuertemente exotérmico. Este hecho puede introducir un factor perturbador en el proceso industrial y sus efectos deberán estudiarse con detalle en una planta piloto.

\subsubsection{Efecto del tamaño de la tobera de inyección de gas de arrastre}

La tobera empleada para la inyección de gas de arrastre se muestra en la figura 3 . El caudal de gas de arrastre variaba con el transcurso del proceso, ya que, como se indica más adelante, la tobera se obstruía con frecuencia. De esta forma, dicho caudal se ajustó continuamente con el propósito de mantener constante la presión de vacío requerida en el reactor.

El carbonato básico precipitado es altamente incrustante por lo que la tobera empleada para inyectar el gas de arrastre se obstruyó con frecuencia. Esto llevó al empleo de una tobera de mayor diámetro, observándose que:

- Al utilizar una tobera de mayor tamaño se retarda el inicio de la precipitación del carbonato básico de cinc. Así, cuando se operó con la tobera fina, se necesitaron $40 \pm 8$ min para que precipitara el $10 \%$ del cinc contenido en la lejía; al trabajar con una tobera de mayor diámetro, dicho proceso se produjo a los $90 \pm 10 \mathrm{~min}$ (a $0,35 \mathrm{~atm}$ de presión, $65^{\circ} \mathrm{C}$ y una concentración inicial de cinc de $80 \pm 5 \mathrm{~g} / \mathrm{l}$ ).

- La velocidad de precipitación del cinc es mayor cuando se utiliza la tobera fina $(0,324 \pm 0,05$ $\mathrm{g} / \mathrm{l} \cdot \mathrm{min})$ que cuando se emplea la de mayor tamaño $(0,17 \pm 0,02 \mathrm{~g} / \mathrm{l} \cdot \mathrm{min})(\mathrm{a} 0,35 \mathrm{~atm}$ de presión, $65^{\circ} \mathrm{C}$ y una concentración inicial de cinc de $80 \pm 5 \mathrm{~g} / \mathrm{l})$.

No se ha podido hallar una relación entre los parámetros estudiados y el diámetro de la tobera, ya que el mismo varía continuamente debido a la formación de una capa de carbonato básico que lo modifica, disminuyendo paulatinamente hasta el taponamiento total.

En la figura 8 se puede ver que, en las condiciones experimentales descritas, tanto la velocidad de precipitación del carbonato básico de cinc como la eliminación de amoniaco y la evaporación de agua es muy superior en el caso de utilizar la tobera fina.

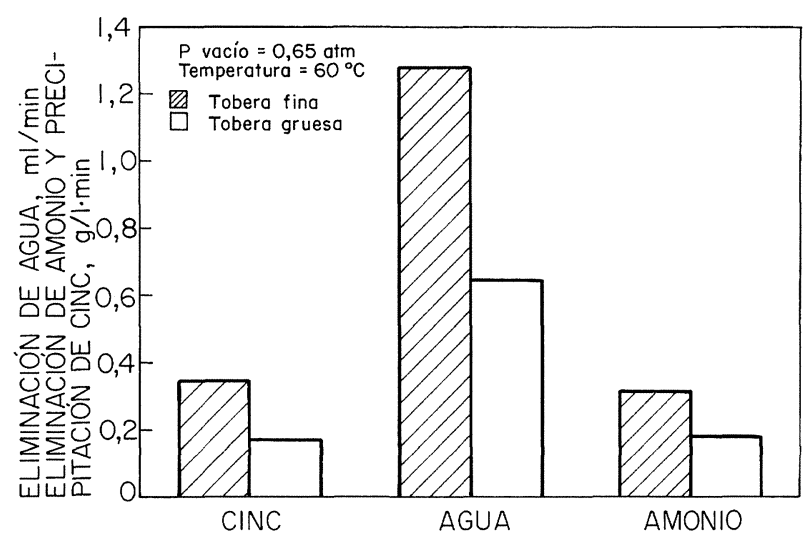

FIG. 8.- Efecto del tamaño de la tobera sobre la velocidad de eliminación de amonio y de agua y sobre la velocidad de precipitación del cinc.

FIG. 8.- Nozzle size influence over ammonium and water elimination velocity and over zinc precipitation velocity. 
En la figura 9 se aprecia que, en las condiciones experimentales indicadas, cuando se utiliza la tobera gruesa es necesario eliminar más agua para que comience a precipitar el carbonato básico. Sin embargo, cuando ha precipitado el $50 \%$ del cinc, se ha eliminado más agua al emplear la tobera fina.

Estos resultados parecen indicar una mayor velocidad de nucleación al utilizar la tobera fina, posiblemente debido al menor tamaño de las burbujas del gas de arrastre.

\subsubsection{Características del carbonato básico de cinc precipitado}

Cuando se ha eliminado el amoniaco libre de la disolución comienza la precipitación del carbonato básico de cinc, en forma de precipitado adherente, del tipo sal incrustante.

En ese instante, inicio de la precipitación del carbonato básico de cinc, precipita completamente el hierro presente en la disolución. De esta forma, cuando ha precipitado un $1 \%$ de cinc, la disolución queda exenta de hierro.

El carbonato básico precipitado es un compuesto no estequiométrico, formado por una mezcla de $\mathrm{ZnCO}_{3}$ y $\mathrm{ZnCO}_{3} \cdot 2 \mathrm{Zn}(\mathrm{OH})_{2}$ en una proporción aproximada de 80 y $20 \%$, respectivamente, y que varía en función de la:

- Composición inicial de la disolución.

- Forma de conducir el proceso.

- Cantidad de cinc precipitado (el precipitado es más rico en cinc al principio de la operación que al final).

Su capacidad incrustante es muy alta, y se deposita preferentemente en las partes calientes del circuito. La facilidad con que se deposita en estos

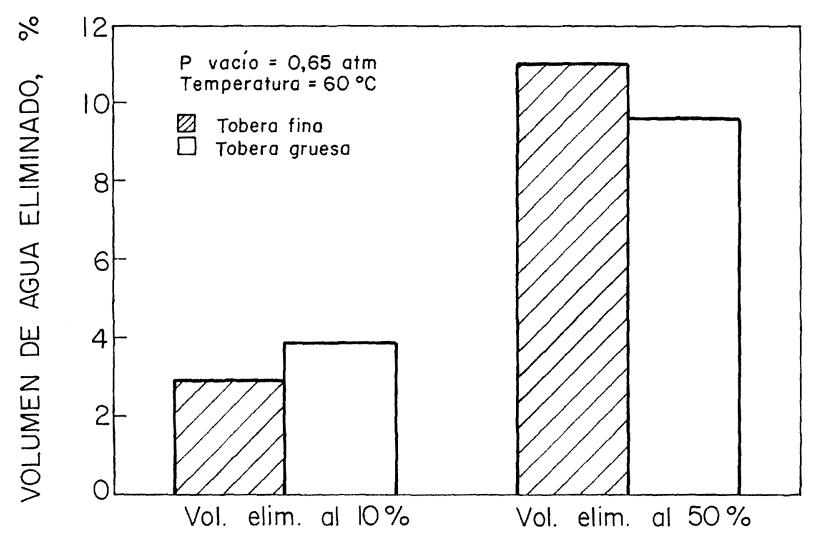

FIG. 9.- Efecto del tamaño de la tobera empleada sobre el volumen de agua eliminado. FIG. 9.- Nozzle size influence over eliminated
water volume. puntos calientes es similar a la de redisolución, tanto en medio ácido como en la propia disolución a precipitar, siempre y cuando exista amonio libre.

En las condiciones óptimas de trabajo, el $90 \%$ del cinc precipita en la primera hora del proceso, y el tiempo de residencia medio para el cual es posible dar por terminada la operación se puede estimar aproximadamente en $90 \mathrm{~min}$. Tras este tiempo la concentración de cinc en la disolución es inferior a $10 \mathrm{~g} / \mathrm{l}$.

La velocidad media de precipitación del cinc es de $1,24 \mathrm{~g} / \mathrm{l} \cdot \mathrm{min}$ con calefacción eléctrica y 1,02 g/l-min cuando se utiliza vapor de agua como gas de arrastre y agente de calefacción.

El carbonato básico precipitado debe poseer unas características físicas que permitan que las operaciones de separación y de lavado se puedan realizar con facilidad. Se estudiaron estas características teniendo en cuenta la filtrabilidad y decantabilidad de las pulpas obtenidas en los diferentes ensayos realizados.

La velocidad de filtración se determinó midiendo el tiempo que tardaban en filtrarse $20 \mathrm{ml}$ de la pulpa extraída del reactor a través de un papel de filtro estándar (papel de filtro plisado de $9 \mathrm{~cm}$ de diámetro).

Para medir la velocidad de sedimentación se introdujeron $50 \mathrm{ml}$ de la pulpa extraída del reactor ciclónico en una probeta de $50 \mathrm{ml}$ graduada y se determinó en cada ensayo la altura de la interfase líquido claro-pulpa frente al tiempo. La densidad se determinó por pesada de un volumen conocido de pulpa. Con estos datos se calculó la velocidad media de sedimentación.

No se han encontrado variaciones significativas de la velocidad de filtración de la pulpa en función de las condiciones de presión y de temperatura a las que se realizó el proceso de precipitación. Por contra, se ha observado que la concentración inicial de cinc en la lejía influye considerablemente sobre dicho parámetro.

Estos mismos efectos se aprecian al determinar la densidad y la velocidad media de decantación a los $30 \mathrm{~min}$, tal y como se puede ver en la tabla VI, donde se presentan los valores medios obtenidos en los diversos ensayos.

\subsubsection{Datos prácticos de la operación de precipitación}

Teniendo en cuenta los ensayos realizados, se pueden hacer las siguientes consideraciones:

- Se puede calentar tanto de forma indirecta, a través de intercambiadores de calor, como directamente mediante inyección de vapor de agua. 
TABLA VI.- Efecto de la concentración inicial de cinc sobre la densidad y sobre la velocidad de sedimentación de la pulpa de precipitación

TABLE VI.- Effect of the initial zinc concentration over precipitation, sedimentation velocity and density of the pulp

\begin{tabular}{|c|c|c|}
\hline $\begin{array}{c}\text { Concentración inicial } \\
\text { de cinc en la lejía, } \\
\mathrm{g} / \mathrm{l}\end{array}$ & $\begin{array}{c}\text { Densidad } \\
\text { pulpa, } \\
\mathrm{g} / \mathrm{cm}^{3}\end{array}$ & $\begin{array}{c}\text { Velocidad media } \\
30 \mathrm{~min}, \\
\mathrm{~m} / \mathrm{min}\end{array}$ \\
\hline$\geq 80$ & 1,95 & 1,12 \\
$\leq 45$ & 1,18 & 0,61 \\
\hline
\end{tabular}

- Las condiciones idóneas de trabajo, en cuanto a presión y temperatura, son las correspondientes al punto de ebullición de la disolución, que aproximadamente corresponden a

$$
\begin{array}{ll}
P & 0,45-0,5 \mathrm{~atm} \text { de presión absoluta } \\
T & 70-75^{\circ} \mathrm{C} .
\end{array}
$$

- Es deseable trabajar con disoluciones que presenten el mayor contenido posible de cinc, y que éste sea siempre superior a $75 \mathrm{~g} / \mathrm{l}$.

- Al evaporarse un $10 \%$ de la disolución finaliza la eliminación del amoniaco libre y comienza la precipitación del cinc.

- $\mathrm{Al}$ evaporarse un $50 \%$ de la disolución se puede dar por terminada la operación de precipitación.

\subsection{Lavado del carbonato básico de cinc}

El carbonato básico de cinc precipitado, una vez seco, se encuentra impurificado por la presencia de ion cloruro procedente de las aguas madres retenidas por dicho compuesto, lo que dificultará su venta y las operaciones posteriores de transformación. Por lo tanto, se hace necesaria su eliminación, lo que se llevó a cabo mediante un lavado con agua.

La pulpa resultante de los ensayos de precipitación se sometió a una separación sólido/líquido mediante sedimentación y filtración a vacío del decantado. El sólido filtrado se lavó con agua en dos etapas a contracorriente con separación intermedia por decantación, tal y como se muestra en la figura 10. La relación agua de lavado/precipitado empleada fue $1 / 1$ en peso.

Operando según el esquema indicado se ha conseguido un carbonato básico de cinc con menos de $0,01 \%$ de $\mathrm{Cl}$.

Dada la cristalinidad y filtrabilidad de este producto sería muy interesante estudiar la operación de lavado mediante la utilización de centrífugas, con el fin de ahorrar agua.

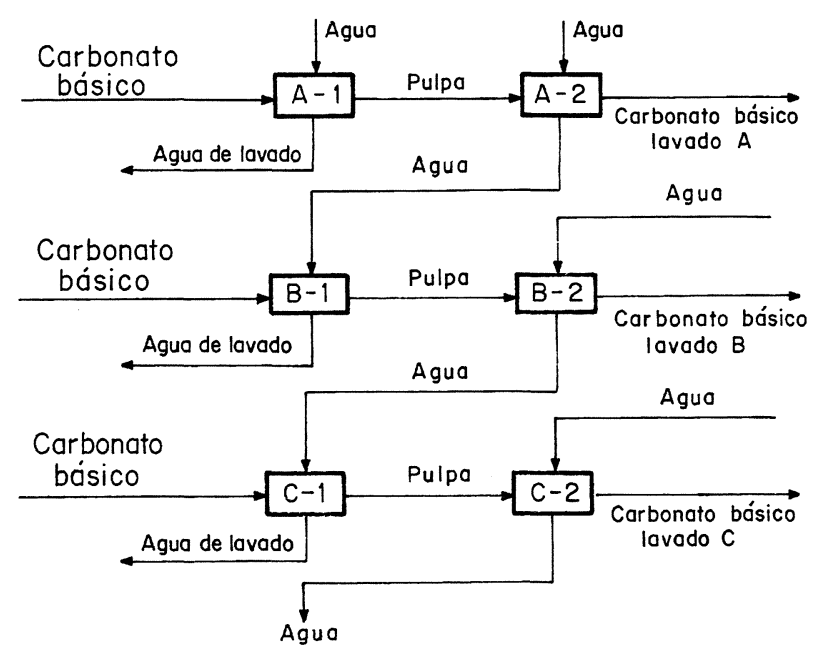

FIG. 10.- Proceso discontinuo de lavado del carbonato básico de cinc precipitado.

FIG. 10.-Batch process for the precipitated zinc basic carbonate washing.

\subsection{Transformación del carbonato básico de cinc}

La falta de constancia en la composición química del carbonato básico provoca que no se pueda ofertar directamente al mercado de consumo, por lo que es preciso transformarlo en otro producto de composición constante con demanda en el mercado.

En la figura 11 se muestran diversas posibilidades de transformación del carbonato básico de cinc. El paso de carbonato básico a cinc metal es sencillo, pues basta disolver el precipitado en el electrólito agotado procedente de la electrólisis, y someter a electrólisis la disolución así obtenida.

La transformación del carbonato básico de cinc en otra sal de cinc (sulfato, cloruro, etc.), se puede realizar disolviendo dicho compuesto en el ácido correspondiente y, posteriormente, cristalizar la sal presente en la disolución.

La transformación en óxido de cinc puede llevarse a cabo fácilmente por calcinación del carbonato básico de cinc. El único problema que se

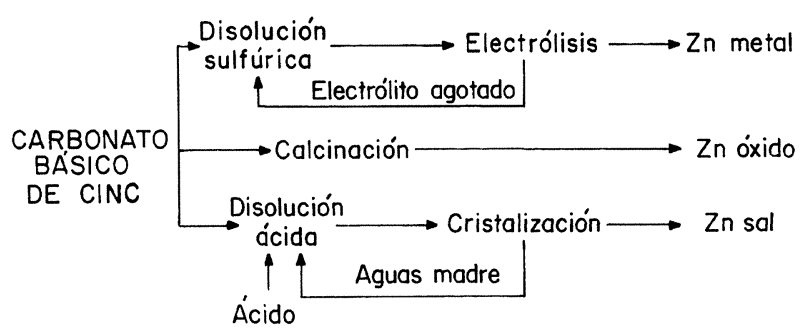

FIG. 11.- Alternativas para la transformación del carbonato básico de cinc.

FIG. 11.- Alternative ways for the zinc basic carbonate transformation. 
puede presentar en este caso es el desconocimiento de la temperatura de descomposición térmica del carbonato básico de cinc obtenido, ya que este producto es un compuesto no estequiométrico del que no existen datos en la bibliografía a tal respecto.

Se determinó la curva de calcinación del carbonato básico. Los resultados obtenidos se representan en la figura 12. Se comprueba que es suficiente un calentamiento a $400-450{ }^{\circ} \mathrm{C}$ para conseguir que el peso de la muestra calcinada permanezca constante. Calcinando durante $1 \mathrm{~h}$ a $425^{\circ} \mathrm{C}$ el carbonato básico lavado, se obtuvo un óxido de cinc con un $79,9 \%$ de cinc y que presenta una superficie específica de $2,64 \mathrm{~m}^{2} / \mathrm{g}$.

\section{COMENTARIOS A LOS RESULTADOS OBTENIDOS Y PROPUESTA DE UN MÉTODO DE TRATAMIENTO DE LOS ÓXIDOS WAELZ}

Tras los ensayos realizados, se propone como método para el tratamiento hidrometalúrgico de los óxidos Waelz el proceso esquematizado en la figura 13.

Con este tratamiento se puede esperar una recuperación de más del $90 \%$ del cinc contenido en los óxidos Waelz, al mismo tiempo que se recuperan el cobre y el cadmio en una proporción próxima al $80 \%$.

La recuperación del cobre y del cadmio se llevará a cabo integrando en el método el proceso COCADEX.

Es posible recuperar el cinc en el estado más conveniente, cinc metal, óxido o sal, con la calidad necesaria para lograr un alto valor económico.

Quedan pendientes por resolver una serie de incógnitas, importantes a la hora de comercializar el proceso, como son:

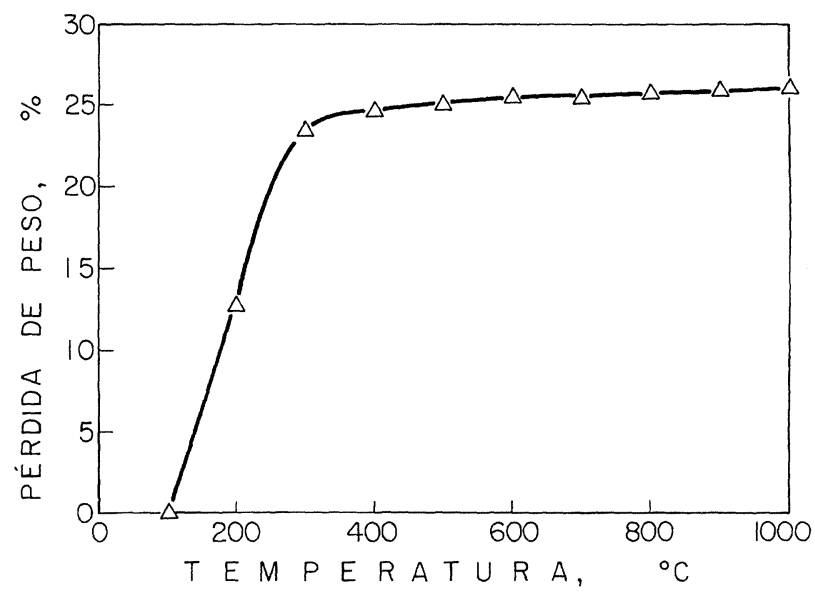

FIG. 12.- Curva de calcinación del carbonato básico de cinc.

FIG. 12.-Zinc basic carbonate calcination graph.

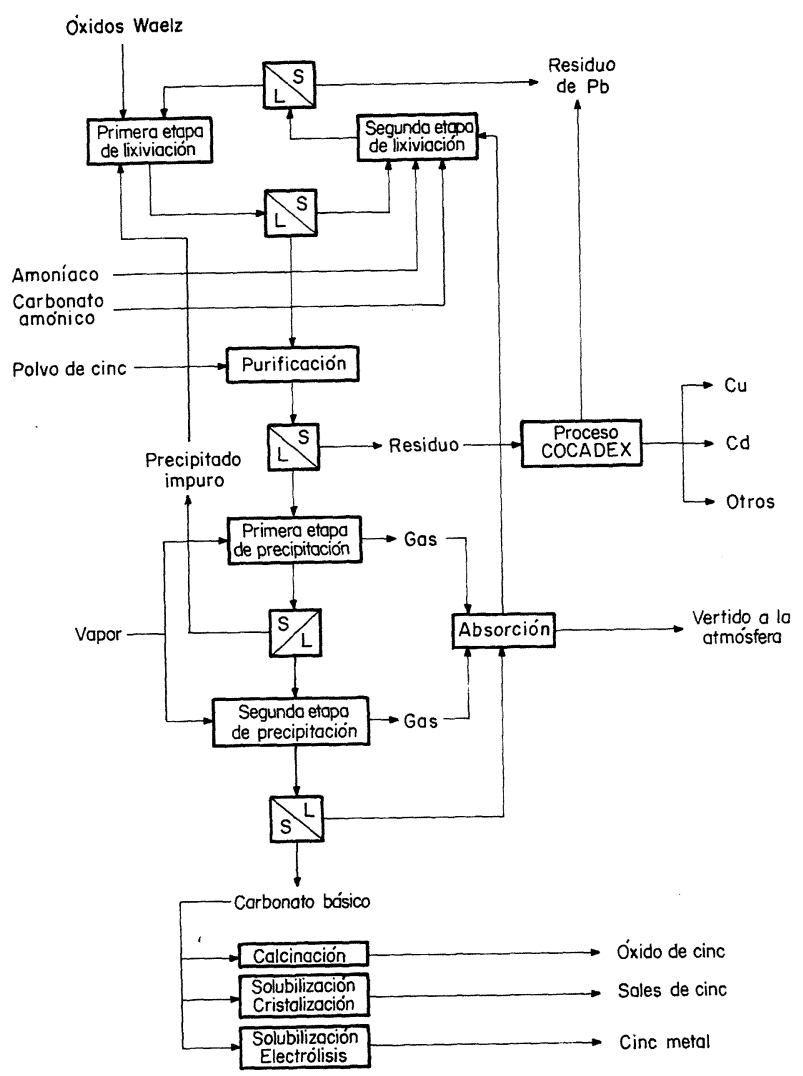

FIG. 13.- Método propuesto para el tratamiento hidrometalúrgico de los óxidos Waelz.

FIG. 13.- Proposed method for the hydrometallurgical treatment of Waelz oxides.

- Recuperación y reciclado del $\mathrm{NH}_{3}$ y $\mathrm{CO}_{2}$.

- Recuperación del plomo contenido en el residuo de lixiviación.

- Método de vertido del residuo final.

- Método para controlar el nivel de cloruros en las lejías.

El primer aspecto está suficientemente estudiado, tanto en el proceso Solvay como en la recuperación del níquel de las lateritas.

En cuanto al segundo problema, existen varias soluciones posibles:

- Retorno al proceso Waelz.

- Tratamiento vía lixiviación con disoluciones de sosa.

- Tratamiento vía lixiviación con disoluciones ácidas cloruradas.

En cuanto al vertido del residuo final, la solución a adoptar dependerá de la solución dada al punto anterior y, por tanto, deberá contemplarse conjuntamente con ésta.

El control de la concentración de ion cloruro en la lejía de lixiviación se puede realizar mediante 
una purga controlada de las aguas madres, donde se encuentra concentrado, y en esta purga precipitar el cinc residual y eliminar el amoniaco mediante adición de cal. El precipitado se retornaría a la etapa de lixiviación y los vapores amoniacales se enviarían a la torre de recuperación de amoniaco. De esta forma, quedaría una disolución de cloruro cálcico que no plantearía problemas para su vertido.

\section{Agradecimiento}

Los autores desean expresar su agradecimiento a la empresa Aser, S.A., en la persona de su Director, D. Néstor Goicochea, por habernos proporcionado los óxidos Waelz utilizados en el desarrollo del presente trabajo.

\section{REFERENCIAS}

(1) Fidalgo, J.M. Proc. IX Cong. Int. de Minería y Metalurgia. Asociación Nacional de Ingenieros de Minas de España. León (España). 1994: 139-166.

(2) AunE, J.A. et al. Recycling of Metalliferous Materials. IMM. 1990: 11-19.

(3) Klein, H. y Bebber, H. Recycling of Metalliferous Materials. IMM. 1990: 125-131.

(4) Piret, N.L. y Castle, J.F. Recycling of Metalliferous Materials. IMM. 1990: 181-219.
(5) Rebollo, J.L. y Faggioni, N. Proc. IX Cong. Int. de Minería y Metalurgia. Asociación Nacional de Ingenieros de Minas de España. León (España). 1994: 79-84.

(6) LimPo, J.L. et al. Procedimiento para la recuperación de cinc, cobre y plomo de minerales y materiales oxidados y/o sulfurados. Patente española núm. 8902487.

(7) Mehlbeer, J. y Melcher, G.A. Proc. Int. Symp. Complex Metallurgy '78. IMM. (R.F.A.). 1978: 96-100.

(8) Diaz, G.; Martin, D. y Lombera, C. $2^{a}$ Int. Conf. on the Recycling of Metals. ASM International. Amsterdam, (Holanda). 1994: 77-84.

(9) Regife, J.M. et al. Ing. Quím., Nov. 1975: 27-33.

(10) Hermana, E. Proc. $7^{\circ}$ Congreso Nacional de Ciencia y Tecnología Metalúrgicas. Asamblea General del CENIM. Madrid (España). 1990: 427-435.

(11) Martin, D. y Hermana, E. Proc. $7^{\circ}$ Cong. Nacional de Ciencia y Tecnología Metalúrgicas. Asamblea General del CENIM. Madrid (España). 1990: 493-502.

(12) De Juan, D.; Perales, A. y Collados, F. Proc. IX Cong. Int. de Minería y Metalurgia. Asociación Nacional de Ingenieros de Minas de España. León (España). 1994: 167-180.

(13) Collados, F. Estudio de las características del par $\mathrm{NH}_{4} \mathrm{OH} / \mathrm{NH}_{4}^{+}$como agente de disolución del $\mathrm{Zn}(\mathrm{II})$. Mecanismo y cinética de la disolución de muestras artificiales (óxidos Waelz). Trabajo Fin de Carrera. Escuela Politécnica Superior de Cartagena (Universidad de Murcia). 1994.

(14) Perales, A. Estudio Técnico Económico de un Método de Tratamiento no Contaminante de los Residuos de Purificación en la Hidrometalurgia del Cinc. Tesis Doctoral. Univ. de Murcia. 1993. 